\title{
ABSTRACT HOMOTOPY THEORY
}

\author{
BY \\ EDGAR H. BROWN, JR.(1)
}

1. Introduction. Let $\mathscr{C}$ be a category and let $\mathscr{S}$ be the category of sets and set maps. If $X$ and $Y$ are objects in $\mathscr{C},[X, Y]$ will denote the set of morphisms from $X$ to $Y$. Let $H: \mathscr{C} \rightarrow \mathscr{S}$ be a contravariant functor. In the case where $\mathscr{C}$ is the category of CW complexes with base point and homotopy classes of maps, conditions on $H$ were given in [1] which implied that $H$ was naturally equivalent to $\left[, Y_{H}\right]$ for some CW complex $Y_{H}$. Furthermore, the proof of this result used, for the most part, abstract category arguments. The aim of this paper is to formalize this latter fact. That is, we wish to give conditions on an abstract category $\mathscr{C}$ and conditions on $H$ from which we can deduce that there is a natural equivalence $T:\left[, Y_{H}\right] \rightarrow H$. Furthermore, we want the category of $\mathrm{CW}$ complexes and homotopy classes of maps to satisfy our conditions on $\mathscr{C}$.

In $\$ 2$ we state and prove our main results. $\$ 3$ contains some examples of categories and functors satisfying our conditions on $\mathscr{C}$ and $H$.

2. Homotopy categories and functors. A pair $\left(\mathscr{C}, \mathscr{C}_{0}\right)$, where $\mathscr{C}$ is a category and $\mathscr{C}_{0}$ is a subcategory, will be called a homotopy category if it satisfies conditions (2.1)-(2.4) below ( $\mathscr{C}_{0}$ plays the role of finite $\mathrm{CW}$ complexes):

(2.1) $\mathscr{C}_{0}$ is a small, full subcategory of $\mathscr{C}$, i.e. $\mathscr{C}_{0}$ is a set and $[X, Y]_{0}=[X, Y]$ if $X$ and $Y \in \mathscr{C}_{0}$.

(2.2) $\mathscr{C}_{0}$ has finite sums and $\mathscr{C}$ has infinite sums.

(2.3) If $f_{i}: A \rightarrow X_{i}, i=1,2$, are in $\mathscr{C}$, there are maps $g_{i}: X_{i} \rightarrow Z$ in $\mathscr{C}$ such that $g_{1} f_{1}=g_{2} f_{2}$ and such that if $g_{i}^{\prime}: X_{i} \rightarrow Z^{\prime}$ satisfy $g_{1}^{\prime} f_{1}=g_{2}^{\prime} f_{2}$, then $g_{i}^{\prime}=h g_{i}$ for some $h: Z \rightarrow Z^{\prime}$. ( $h$ is not necessarily unique.) Furthermore, if $A$ and $X_{i} \in \mathscr{C}_{0}$, $Z$ may be chosen in $\mathscr{C}_{0}$. ( $Z$ plays the role of $X_{1} \cup X_{2}$ where $X_{1} \cap X_{2}=A$.)

(2.4) If $f_{n}: X_{n} \rightarrow X_{n+1}, n=1,2,3, \cdots$, are in $\mathscr{C}$ there is an $X \in \mathscr{C}$ and maps $g_{n}: X_{n} \rightarrow X$ such that:

(i) $\operatorname{dir} \lim g_{n *}: \operatorname{dir} \lim [Z, X] \approx[Z, X]$ for $Z \in \mathscr{C}_{0}, g_{n *}$ denotes the map $g_{n *}(h)=g_{n} h$.

(ii) inv $\lim g_{n}^{*}:[X, Z] \rightarrow \operatorname{inv} \lim \left[X_{n}^{*}, Z\right]$ is an epimorphism for all $Z \in \mathscr{C}$. $g_{n}^{*}(h)=h g_{n}$.

Let $\hat{\mathscr{C}}$ be the category of $\mathrm{CW}$ complexes and homotopy classes of maps. Let

Received by the editors June 8, 1964.

(1) The results in this paper were obtained while the author was on an NSF Fellowship. 
$\hat{\mathscr{C}}_{0}$ be the full subcategory of $\mathscr{C}$ whose objects are the finite subcomplexes of some standard countably infinite dimensional simplicial complex.

THEOREM 2.5. $\left(\hat{\mathscr{C}}, \hat{\mathscr{C}}_{0}\right)$ is a homotopy category.

Proof. (2.1) and (2.2) are immediate. For (2.3) we construct $g_{i}$ and $Z$ as follows:

$$
Z=A \times I \cup X_{1} \cup X_{2}
$$

where $(a, 0)$ is identified to $f_{1}(a)$ and $\left(a_{1}, 1\right)$ is identified to $f_{2}(a)$ for all $a \in A$. $g_{i}$ is the homotopy class of the inclusion map of $X_{i}$ into $Z$. (2.4)(i) follows from the fact that a compact subset of a CW complex is contained in a finite subcomplex. (2.4) (ii) follows from the homotopy extension theorem.

If $\left(\mathscr{C}, \mathscr{C}_{0}\right)$ is a homotopy category and $H: \mathscr{C} \rightarrow \mathscr{S}$ is a contravariant functor, $H$ will be called a homotopy functor if it satisfies conditions (2.6) and (2.7) below:

(2.6) If $h_{\alpha}: X_{\alpha} \rightarrow \sum X_{\beta}$ are the injections,

$$
\prod H\left(h_{\alpha}\right): H\left(\Sigma X_{\alpha}\right) \approx \prod H\left(X_{\alpha}\right) \text {. }
$$

(2.7) If $f_{i}: A \rightarrow X_{i}$ and $g_{i}: X_{i} \rightarrow Z, i=1,2$, are as in (2.3) and $u_{i} \in H\left(X_{i}\right)$ satisfy $H\left(f_{1}\right) u_{1}=H\left(f_{2}\right) u_{2}$, then there is a $v \in H(Z)$ such that $H\left(g_{i}\right) v=u_{i}$.

REMARK. It is immediate that $[, X], X \in \mathscr{C}$, is a homotopy functor.

If $\left(\mathscr{C}, \mathscr{C}_{0}\right)$ is a homotopy category, $\overline{\mathscr{C}}_{0}$ will denote the set of objects $Y \in \mathscr{C}$ such that $f: Y \rightarrow Y^{\prime}$ is an equivalence if $f_{*}:[X, Y] \approx\left[X, Y^{\prime}\right]$ for all $X \in \mathscr{C}_{0}$. For example, if $\mathscr{C}$ is the category of $\mathrm{CW}$ complexes with base point and homotopy classes of maps and $\mathscr{C}_{0}$ is the subcategory of finite simplicial complexes as above, then $\overline{\mathscr{C}}_{0}$ is the set of all connected $\mathrm{CW}$ complexes.

THEOREM 2.8. If $H$ is a homotopy functor, there is $a Y_{H} \in \mathscr{C}$ and a natural transformation $T:\left[, Y_{H}\right] \rightarrow H$ such that $T:\left[X, Y_{H}\right) \approx H(X)$ for all $X \in \mathscr{C}_{0}$. Furthermore, if $Y_{H} \in \overline{\mathscr{C}}_{0}, Y_{H}$ is unique up to an equivalence and $T:\left[X, Y_{H}\right] \approx H(X)$ for all $X \in \mathscr{C}$.

REMARK. In applications of (2.8) one can sometimes deduce that $Y_{H} \in \overline{\mathscr{C}}_{0}$ from the properties of $H$. For example, if $\mathscr{C}$ is the category of $\mathrm{CW}$ complexes with base point as above, $Y_{H} \in \overline{\mathscr{C}}_{0}$ if $H\left(S^{0}\right)$ contains only one element.

THEOREM 2.9. If $\mathscr{C}_{0}$ is a countable set, $H: \mathscr{C}_{0} \rightarrow \mathscr{S}$ is a contravariant functor satisfying (2.6) for finite sums and (2.7) and if $H(X)$ is countable for each $X \in \mathscr{C}_{0}$, then $H$ can be extended to a homotopy functor $\bar{H}: \mathscr{C} \rightarrow \mathscr{S}$.

Before proving (2.8) and (2.9) we give some lemmas.

LEMMA 2.10. If (2.2) holds, (2.3) is equivalent to the following: If $f_{1}, f_{2}: A \rightarrow X$, there is a $g: X \rightarrow Z$ such that $g f_{1}=g f_{2}$ and if $g^{\prime}: X \rightarrow Z^{\prime}$ satisfies $g^{\prime} f_{1}=g^{\prime} f_{2}$, 
then $g^{\prime}=h g$ for some $h: Z \rightarrow Z^{\prime}$. Furthermore if $A$ and $X \in \mathscr{C}_{0}, Z$ may be chosen in $\mathscr{C}_{0}$.

Proof. If (2.3) holds, take $g: X \rightarrow Z$ to be the map given by (2.3) from the pair $f_{1}+f_{2}: A+A \rightarrow X$ and $i d+i d: A+A \rightarrow A$. If the above condition holds, take $g_{i}: X_{i} \rightarrow Z$ to be $g k_{i}$, where $k_{i}: X_{i} \rightarrow X_{1}+X_{2}$ are the injections and $g: X_{1}+X_{2} \rightarrow Z$ comes from $k_{1} f_{1}, k_{2} f_{2}: A \rightarrow X_{1}+X_{2}$.

We will call the map $g: X \rightarrow Z$ as in (2.10) an equalizer $\left({ }^{2}\right)$ of $f_{1}$ and $f_{2}$.

LEMMA 2.11. If $H$ is a homotopy functor, $g: X \rightarrow Z$ is an equalizer of $f_{1}, f_{2}: A \rightarrow X$ and $u \in H(X)$ is such that $H\left(f_{1}\right) u=H\left(f_{2}\right) u$, then there is $a$ $v \in H(Z)$ such that $H(g) v=u$.

Proof. This is immediate from (2.6) and the proof of (2.10).

Lemma 2.12. If $f_{n}: X_{n} \rightarrow X_{n+1}$ and $g_{n}: X_{n} \rightarrow X$ are as in (2.4), then inv $\lim H\left(g_{n}\right): H(X) \rightarrow \operatorname{inv} \lim H\left(X_{n}\right)$

is an epimorphism.

Proof. $X$ is an equalizer of $\sum i d_{n}, \Sigma f_{n}: \Sigma X_{n} \rightarrow \Sigma X_{n}$. Since $\prod H\left(X_{n}\right) \approx H\left(\Sigma X_{n}\right)$,

$$
\text { inv } \lim H\left(X_{n}\right) \approx\left\{u \in H\left(\sum X_{n}\right) \mid H\left(\sum i d_{n}\right) u=H\left(\sum f_{n}\right) u\right\} \text {. }
$$

(2.12) now follows from (2.11).

Proof of Theorem 2.8. Note that if $u \in H(Y), T_{u}:[X, Y] \rightarrow H(X)$, where $T_{u}(f)=H(f) u$, is a natural transformation.

If $\gamma$ is anything and $X \in \mathscr{C},(X, \gamma) \in \mathscr{C}$ will denote a copy of $X$ and $t_{\gamma}:(X, \gamma) \rightarrow X$ will be an equivalence. In the following we will identify $H\left(\sum X_{\alpha}\right)$ and $\prod H\left(X_{\alpha}\right)$.

We define, by induction on $n, Y_{n} \in \mathscr{C}, u_{n} \in H\left(Y_{n}\right)$ and $f_{n}: Y_{n} \rightarrow Y_{n+1}$ for $n=0,1,2, \cdots$. Let $Y_{0}$ and $u_{0}$ be arbitrary. Let

$$
Y_{1}=Y_{0}+\Sigma(X, u)
$$

where the sum ranges over $X \in \mathscr{C}_{0}$ and $u \in H(X)$. Let $u_{1}=\left(u_{0}, \cdots, H\left(t_{u}\right) u, \cdots\right)$ and let $f_{1}$ be the injection. Suppose $Y_{n}$ and $u_{n}, n \geqq 1$, have been defined. Let $f_{n}: Y_{n} \rightarrow Y_{n+1}$ be the equalizer of

$$
\sum g_{1} t_{\left(g_{1}, g_{2}\right)}, \quad \sum g_{2} t_{\left(g_{1}, g_{2}\right)}: \Sigma\left(X,\left(g_{1}, g_{2}\right)\right) \rightarrow Y_{n}
$$

where the sum ranges over all $X \in \mathscr{C}_{0}$ and pairs $g_{1}, g_{2}: X \rightarrow Y_{n}$ such that $g_{1} \neq g_{2}$ and $H\left(g_{1}\right) u_{n}=H\left(g_{2}\right) u_{n}$. By (2.11) there is a $u_{n+1} \in H\left(Y_{n+1}\right)$ such that $H\left(f_{n}\right) u_{n+1}=u_{n}$.

From the way in which the $Y_{n}$ 's were constructed, it follows that

(2) (2.10) and a more complete discussion of equalizers appears in [5]. 


$$
\operatorname{dir} \lim T_{u_{n}}: \operatorname{dir} \lim \left[X, Y_{n}\right] \approx H(X)
$$

for $X \in \mathscr{C}_{0}$.

By (2.4) and (2.12), there is a $Y_{H} \in \mathscr{C}$, maps $g_{n}: Y_{n} \rightarrow Y_{H}$ and $u \in H\left(Y_{H}\right)$ such that $H\left(g_{n}\right) u=u_{n}$ and

$$
\operatorname{dir} \lim g_{n_{*}}: \operatorname{dir} \lim \left[X, Y_{n}\right] \approx\left[X, Y_{H}\right]
$$

for $X \in \mathscr{C}_{0}$. One may easily check that $\operatorname{dir} \lim T_{u_{n}}=T_{u} \operatorname{dir} \lim g_{n *}$. Hence

$$
T_{u}:\left[X, Y_{H}\right] \approx H(X)
$$

for all $X \in \mathscr{C}_{0}$.

Suppose $Y_{H} \in \overline{\mathscr{C}}_{0}$. We wish to show that $T_{u}:\left[X, Y_{H}\right] \approx H(X)$ for all $X \in \mathscr{C}$. Suppose $f_{1}, f_{2}: X \rightarrow Y_{H}$ are such that $T_{u}\left(f_{1}\right)=T_{u}\left(f_{2}\right)$. Then $H\left(f_{1}\right) u=H\left(f_{2}\right) u$. Let $g: Y_{H} \rightarrow Z$ be the equalizer of $f_{1}$ and $f_{2}$ and let $v \in H(Z)$ be such that $H(g) v=u$. Recall that in the construction of $Y_{n}$ and $u_{n}$ above $Y_{0}$ and $u_{0}$ were arbitrary. Repeat the above construction of $Y_{n}$ and $u_{n}$ starting with $Y_{0}=Z, u_{0}=v$ to obtain $Y_{H}^{\prime}, u^{\prime}$ and $h: Z \rightarrow Y_{H}^{\prime}$ such that $H(h) u^{\prime}=v$ and $T_{u^{\prime}}:\left[X, Y_{H}^{\prime}\right] \approx H(X)$ for for $X \in \mathscr{C}_{0}$. Then $H(h g) u^{\prime}=u$ and hence $T_{u^{\prime}}(h g)_{*}=T_{u}$. Therefore $(h g)_{*}:\left[X, Y_{H}\right] \approx\left[X, Y_{H}^{\prime}\right]$ for $X \in \mathscr{C}_{0}$. Since $Y_{H} \in \overline{\mathscr{C}}_{0}, h g$ is an equivalence. But $h g f_{1}=h g f_{2}$. Therefore $f_{1}=f_{2}$.

Suppose $w \in H(X), X \in \mathscr{C}$. Let $i_{1}$ and $i_{2}$ be the injections of $X$ and $Y_{H}$ into $X+Y_{H}$. Repeat the above construction starting with $Y_{0}=X+Y_{H}$ and $u_{0}=(w, u)$ to obtain $Y_{H}^{\prime}, h: X+Y_{H} \rightarrow Y_{H}^{\prime}$ and $u^{\prime} \in H\left(Y_{H}^{\prime}\right)$ such that $H(h) u^{\prime}=(w, u)$. Then, as above, $h i_{2}$ is an equivalence. Let $f=\left(h i_{2}\right)^{-1} h i_{1}$. Then

$$
\begin{aligned}
T_{u}(f) & =H(f) u \\
& =H\left(i_{1}\right) H(h) H\left(h i_{2}\right)^{-1} u \\
& =H\left(i_{1}\right) H(h) u^{\prime} \\
& =w .
\end{aligned}
$$

Therefore $T_{u}:\left[X, Y_{H}\right] \approx H(X)$ for all $X \in \mathscr{C}$.

The uniqueness of $Y_{H}$ up to an equivalence is immediate.

Proof of Theorem 2.10. To prove (2.10) we construct a $Y_{H} \in \mathscr{C}$ and a natural equivalence $T:\left[X, Y_{H}\right] \approx H(X)$ which is defined for all $X \in \mathscr{C}_{0} .\left[, Y_{H}\right]$ then extends $H$ to $\mathscr{C}$. To construct $Y_{H}$ we alter the construction of $Y_{n}$ and $u_{n}$ in the proof of (2.9) so that $Y_{n} \in \mathscr{C}_{0}$ for all $n$. Let $X_{i}$ and $h_{j}, i, j=1,2, \cdots$, be the objects and maps in $\mathscr{C}_{0}$. Let $H\left(X_{i}\right)=\left\{u_{i j}\right\}$. Let $Y_{0} \in \mathscr{C}_{0}$ and $u_{0} \in H\left(Y_{0}\right)$ be arbitrary. Suppose $Y_{k}, u_{k} \in H\left(Y_{k}\right)$ and $f_{k-1}: Y_{k-1} \rightarrow Y_{k}$ have been defined for $k \leqq n$. If $k \leqq l$, let

$$
\begin{aligned}
f_{k l} & =f_{l-1} f_{l-2} \cdots f_{k}, & & l<k, \\
& =i d, & & l=k .
\end{aligned}
$$


Let

$$
\begin{aligned}
& Y_{n+1}^{\prime}=Y_{n}+\sum_{i, j \leqq 1}\left(X_{i}, u_{i j}\right), \\
& u_{n+1}^{\prime}=\left(u_{n}, \cdots, H\left(t_{u_{i j}}\right) u_{i j}, \cdots\right),
\end{aligned}
$$

and let $r: Y_{n} \rightarrow Y_{n+1}^{\prime}$ be the injection. Let $g: Y_{n+1}^{\prime} \rightarrow Y_{n+1}$ be the equalizer of

$$
\sum g_{1} t_{\left(g_{1}, g_{2}\right)}, \sum g_{2} t_{\left(g_{1}, g_{2}\right)}: \Sigma\left(X,\left(g_{1}, g_{2}\right)\right) \rightarrow Y_{n+1}^{\prime}
$$

where $X \in \mathscr{C}_{0}$ and $\left(g_{1}, g_{2}\right)$ ranges over all pairs of the form $g_{1}=r f_{n, k} h_{i}$ and $g_{2}=r f_{n, i} h_{j}$ such that $i, j \leqq n$ and $H\left(g_{1}\right) u_{n+1}^{\prime}=H\left(g_{2}\right) u_{n+1}^{\prime}$. Let $f_{n}=g r$. By (2.11) there is a $u_{n+1} \in H\left(Y_{n+1}\right)$ such that $H\left(f_{n}\right) u_{n+1}=u_{n}$. One may easily check that

$$
\operatorname{dir} \lim T_{u_{n}}: \operatorname{dir} \lim \left[X, Y_{n}\right] \approx H(X)
$$

for all $X \in \mathscr{C}_{0}$. By (2.4) there is a $Y_{H} \in \mathscr{C}$ and maps $g_{n}: Y_{n} \rightarrow Y_{H}$ such that

$$
\operatorname{dir} \lim T_{n^{*}}: \operatorname{dir} \lim \left[X, Y_{n}\right] \approx\left[X, Y_{H}\right]
$$

for $X \in \mathscr{C}_{0}$. Let $T:\left[X, Y_{H}\right] \rightarrow H(X)$ be defined by $T=\left(\operatorname{dir} \lim T_{u_{n}}\right)\left(\operatorname{dir} \lim g_{n *}\right)^{-1}$. It is trivial to check that $T$ is a natural transformation for $X \in \mathscr{C}_{0}$.

REMARK. Suppose $\mathscr{C}$ is the category of $\mathrm{CW}$ complexes with base point and homotopy classes of maps. In this case the construction of the $Y_{n}$ 's for the proofs of (2.9) and (2.10) could have been carried out using only spheres, instead of all the objects in $\mathscr{C}_{0}$. The procedure would then be the usual process of building a CW complex by attaching cells.

\section{Examples of homotopy categories and functors.}

EXAMPLE 3.1. Suppose $\mathscr{C}$ is the category of CW complexes with base point and homotopy classes of maps. Let $\mathscr{C}_{0}$ be the full subcategory of $\mathscr{C}$ whose objects are all finite subcomplexes with base point of some infinite dimensional simplicial complex. As in the proof of $(2.5)$, one may easily verify that $\left(\mathscr{C}, \mathscr{C}_{0}\right)$ is a homotopy category. $\overline{\mathscr{C}}_{0}$ is the class of all connected $\mathrm{CW}$ complexes with base point. In each of the examples below $H\left(S^{0}\right)$ contains exactly one element so that $Y_{H} \in \overline{\mathscr{C}}_{0}$.

Let $\left\{H^{q}, \delta^{q}\right\}$ be a cohomology theory on pairs of $\mathrm{CW}$ complexes [1], i.e. $H^{q}$ and $\delta^{q}$ satisfy all the Eilenberg-Steenrod axioms except the dimension axiom. Furthermore, suppose that if $X=\bigcup X_{\alpha}$ is a disjoint union and $i_{\alpha}: X_{\alpha} \rightarrow X$ is the inclusion map,

$$
\prod H^{q}\left(i_{\alpha}\right): H^{q}(X) \approx \prod H^{q}\left(X_{\alpha}\right) .
$$

Then for each $q$, the following is a homotopy functor:

$$
\begin{gathered}
H\left(X, x_{0}\right)=H^{q}\left(X, x_{0}\right), \\
H([f])=H^{q}(f),
\end{gathered}
$$

where $x_{0}$ is the base point of $X$ and $[f]$ denotes the homotopy class of $f$. 
REMARK. $H^{q}(X, A)$ can be recovered from $H$ as follows: Let $p$ be a point disjoint from $X$ and let $Z \in \mathscr{C}$ be $X \cup(A \times I) \cup\{p\}$ with $(a, 0)$ identified to $a$ and $(a, 1)$ identified to $p$ for each $a \in A$. Let $p$ be the base point of $Z$. Then $H^{q}(X, A)=H(Z)$.

Let $F$ be a topological space and let $G$ be a topological group which acts effectively on $F$. Let $\mathscr{B}_{0}$ be the fibre bundle with fibre $F$, group $G$ and base space a point $p$. We define a bundle over $X \in \mathscr{C}$ to be a pair $(\mathscr{B}, i)$ where $\mathscr{B}$ is a bundle over $X$ in the sense of Steenrod [4] and $i: \mathscr{B}_{0} \rightarrow \mathscr{B}$ is a bundle map which carries $p$ into the base point of $X$. Bundles $\left(\mathscr{B}^{\prime}, i^{\prime}\right)$ and $(\mathscr{B}, i)$ over $X$ will be called equivalent if there is a bundle map $\lambda: \mathscr{B}^{\prime} \rightarrow \mathscr{B}$ over the identity map of $X$ such that $i=\lambda i^{\prime}$. For each $X \in \mathscr{C}$, let $H(X)$ be the set of equivalence classes of bundles over $X$ with fibre $F$ and group $G$. If $f: X \rightarrow Y$ let $H([f]): H(Y) \rightarrow H(X)$ be defined by $H([f])\{\mathscr{B}\}=\left\{f^{*} \mathscr{B}\right\} . H$ is then a homotopy functor. One can recover the usual theorem concerning the existence of a universal bundle from Theorem 2.8 by considering $H\left(X^{+}\right)$where $X^{+}$is $X$ plus a disjoint base point.

Treating the base point as above, the functor which assigns to each $X \in C$ the set of fibre homotopy equivalence classes of fibre spaces over $X$ with fibre $F$ is a homotopy functor. The proof that this functor satisfies $(2.7)$ is by no means trivial but it can be proved by the techniques in [3].

EXAMPLE 3.2. Let $\mathscr{C}$ be the following category. The objects of $\mathscr{C}$ are pairs $(\mathscr{B}, i)$ where $\mathscr{B}$ is a bundle over a CW complex with base point with fibre $F$ and group $G$ and $i: \mathscr{B}_{0} \rightarrow \mathscr{B}$ as above. The maps of $\mathscr{C}$ are homotopy classes of bundle maps respecting our base point convention. Let $\mathscr{C}_{0}$ be the subcategory of bundles over the finite subcomplexes of some infinite dimensional simplicial complex. Then $\left(\mathscr{C}, \mathscr{C}_{0}\right)$ is a homotopy category and the following is a homotopy functor:

$$
H((\mathscr{B}, i))=H^{q}(E, i F)
$$

where $E$ is the total space of $\mathscr{B}$ and $H^{q}$ is a cohomology group as in (3.1). If $F=R^{n}$ and $G=O(n)$, the following is also homotopy functor:

$$
H((\mathscr{B}, i))=H^{q}\left(T(\mathscr{B}), T(i)\left(T\left(\mathscr{B}_{0}\right)\right)\right)
$$

where $T$ is the Thom space functor.

EXAMPLE 3.3. The following is a homotopy category. The objects of $C$ are $n$-tuples $\left(A_{1}, A_{2}, \cdots, A_{n}\right)$ where $A_{1}$ is a $\mathrm{CW}$ complex and $A_{n} \subset A_{n-1} \subset \cdots \subset A_{1}$ are subcomplexes. The maps of $\mathscr{C}$ are homotopy classes of maps in the usual sense. The objects of $\mathscr{C}_{0}$ are $n$-tuples where $A_{1}$ is a finite subcomplex of an infinite- $n$ dimensional simplicial complex.

REMARK. Although Theorem 2.8 cannot be applied to microbundles [2] to prove the existence of universal microbundles, the techniques used to prove (2.8) can be applied in a straightforward manner to obtain this result. The complicating factor is that microbundles are defined only over finite-dimensional base spaces. 
This complication could be introduced into our abstract theory but the result would be more intricate than the method of proof used in (2.8).

\section{BIBLIOGRAPHY}

1. E. H. Brown, Jr., Cohomology theories, Ann. of Math. 75 (1962), 467-484.

2. J. Milnor, Microbundles and differentiable structures, Princeton University, 1961, mimeographed.

3. S. Stasheff, A classification theorem for fibre spaces, Topology 2 (1963), 239-246.

4. N. Steenrod, The topology of fibre bundles, Princeton Mathematical Series, Vol. 14, Princeton Univ. Press, Princeton, N.J., 1951.

5. B. Eckmann and P. J. Hilton, Unions and intersections in homotopy categories, Comment. Math. Helv. 38 (1964), 293-307.

BRANDEIS UNIVERSITY,

Waltham, Massachusetts 